\title{
ON MODIFIED KIES DISTRIBUTION AND ITS APPLICATIONS
}

\author{
C. SATHEESH KUMAR ${ }^{\star}$ \\ Department of Statistics, University of Kerala, Trivandrum-695 581, India \\ Email: drcsatheeshkumar@gmail.com \\ S. H. S. DHARMAJA \\ Department of Statistics, Govt. College for Women, Trivandrum-695 014, India \\ Email: shs_dharmaja@yahoo.com
}

\begin{abstract}
SUMMARY
In this paper, we consider a class of bathtub-shaped hazard function distribution through modifying the Kies distribution and investigate some of its important properties by deriving expressions for its percentile function, raw moments, stress-strength reliability measure etc. The parameters of the distribution are estimated by the method of maximum likelihood and discussed some of its reliability applications with the help of certain real life data sets. In addition, the asymptotic behavior of the maximum likelihood estimators of the parameters of the distribution is examined by using simulated data sets.
\end{abstract}

Keywords and phrases: Failure rate; Fisher information matrix; Maximum likelihood estimation; Model selection; Percentile measures; Simulation.

\section{Introduction}

The Weibull distribution and its extended models have found wide applications in almost all areas of sciences especially in engineering sciences, hydrological sciences, meteorological sciences, social sciences etc. For details of some of these applications, see Meekar and Escobar (1998), Murthy et al. (2004), Rinne (2009) and references therein. Some well-known extended Weibull models studied in the literature are the beta Weibull distribution (BWD) (see Almalki and Nadarajah, 2014; Cordeiro et al., 2013; Famoye et al., 2005), the beta generalized Weibull distribution (BGWD) (see Singla et al., 2012) and the exponentiated Weibull distribution (EWD) (see Mudholkar et al., 1995). All these families of distributions possess increasing, decreasing and/or bathtub-shaped hazard rate functions. Kumar and Dharmaja (2014) studied the Kies distribution (KD) as an alternative to these extended Weibull models and shown that it gives better fit to certain real life data sets compared to both the BWD and the BGWD.

Consider the the following probability density function (pdf) of Kies distribution, in which $0 \leq$ $x \leq \alpha<\infty, \lambda>0$ and $\beta>0$.

$$
g_{1}(x)=g_{1}(x ; \alpha, \lambda, \beta)=\alpha \lambda \beta x^{\beta-1} \exp \left\{-\lambda(x /(\alpha-x))^{\beta}\right\} /(\alpha-x)^{\beta+1}
$$

\footnotetext{
* Corresponding author
}

(C) Institute of Statistical Research and Training (ISRT), University of Dhaka, Dhaka 1000, Bangladesh. 
A distribution with pdf (1.1) hereafter we denote as $K D(\alpha, \lambda, \beta)$. The cumulative distribution function (cdf) $G_{1}(x)$ of the $K D(\alpha, \lambda, \beta)$ is given by

$$
G_{1}(x)=1-\exp \left\{-\lambda(x /(\alpha-x))^{\beta}\right\}, \text { for } x \in(0, \alpha) .
$$

Recently exponentiated type distributions have received much attention in the literature due to its flexibility in modelling certain applications. Analogous to the EWD model, through this paper we propose an exponentiated form of the $K D(\alpha, \lambda, \beta)$ as a bathtub-shaped hazard function distribution and name it as "the modified Kies distribution (MKD)", which can be viewed as a generalization of the exponentiated reduced Kies distribution, $\operatorname{ERK} D(\beta, \delta)$, of Kumar and Dharmaja (2016). We study several important properties of the MKD in Section 2 and it is important to note that the MKD possess increasing or decreasing or bathtub-shaped hazard functions depending on the parameters of the distribution. The maximum likelihood estimation of the parameters of the distribution have been discussed in Section 3 and certain real life data applications in reliability studies are considered in Section 4 for illustrating the usefulness of the proposed class of distributions and also compared the proposed model with the existing models based on fitted values of the distribution and Weibull probability plots. Further, in Section 5 we examine the asymptotic behavior of the maximum likelihood estimators of the parameters of the distribution by using certain simulated data sets.

We need the following integral/series representations in the sequel. For details regarding these representations see Gradshteyn and Ryhzik (2007). For $\operatorname{Re}(\nu)>0, \operatorname{Re}(\mu)>0$,

$$
\int_{0}^{u} x^{\nu-1} \exp (-\mu x) d x=\mu^{-\nu} \gamma(\nu, \mu u)
$$

in which

$$
\begin{gathered}
\gamma(a, u)=\sum_{i=0}^{\infty} \frac{(-1)^{i}}{i !} \frac{u^{a+i}}{a+i}, \\
\int_{0}^{\infty} x^{\nu-1} \exp (-\mu x) d x=\mu^{-\nu} \Gamma(\nu), \\
\int_{u}^{\infty} x^{-\nu} \exp (-x) d x=u^{-\nu / 2} \exp (-\nu / 2) W_{-\frac{\nu}{2},\left(\frac{1-\nu}{2}\right)}(u),
\end{gathered}
$$

and for $|\arg (-x)|<3 \pi / 2$

$$
W_{k_{1}, k_{2}}(x)=\frac{\Gamma\left(-2 k_{2}\right)}{\Gamma\left(\frac{1}{2}-k_{2}-k_{1}\right)} M_{k_{1}, k_{2}}(x)+\frac{\Gamma\left(2 k_{2}\right)}{\Gamma\left(\frac{1}{2}+k_{2}-k_{1}\right)} M_{k_{1},-k_{2}}(x)
$$

with

$$
M_{k_{1}, k_{2}}(x)=\exp (-x / 2) x^{k_{2}+\frac{1}{2}} \sum_{n=0}^{\infty}\left\{\frac{\left(\frac{1}{2}-k_{1}+k_{2}\right)_{n}}{\left(1+2 k_{2}\right)_{n}} \cdot \frac{x^{n}}{n !}\right\},
$$

where $(c)_{n}=c(c+1) \cdots(c+n-1)$, for $n \geq 1$ and $(c)_{0}=1$.

We need the following series representations in the sequel. For any real valued function $A(\cdot, \cdot)$,

$$
\sum_{s=0}^{\infty} \sum_{r=0}^{\infty} A(r, s)=\sum_{s=0}^{\infty} \sum_{r=0}^{s} A(r, s-r) .
$$


(cf. Kumar and Nair, 2012). For $|x|<1$, the following expansion of $(1-x)$ in which ${ }^{\vartheta} C_{r}=$ $\frac{\vartheta !}{r !(\vartheta-r) !}$, for any $\vartheta \geq r$ with $r=0,1,2, \ldots, \vartheta$ and $\vartheta \in N$, the set of all positive integers and $[\vartheta]_{j}=\frac{\Gamma(\vartheta+1)}{\Gamma(\vartheta+1-j)}$, for $\vartheta \in[0, \infty)-N$, for each $j=0,1,2, \ldots$

$$
\begin{aligned}
& (1-x)^{\vartheta}=\sum_{j=0}^{\infty} \frac{(-1)^{j} \Gamma(\vartheta+1)}{j ! \Gamma(\vartheta+1-j)} x^{j}=\left\{\begin{array}{l}
\sum_{j=0}^{\vartheta}(-1)^{j}{ }^{\vartheta} C_{j} x^{j}, \vartheta \in N \\
\sum_{j=0}^{\infty} \frac{(-1)^{j}[\vartheta+1]_{j}}{j !} x^{j}, \vartheta \in R^{+}-N
\end{array}\right. \\
& \operatorname{Ei}(x)= \begin{cases}\gamma+\ln (-x)+\sum_{k=1}^{\infty} \frac{x^{k}}{k \times k !}, & \text { for } x<0 \\
\gamma+\ln (x)+\sum_{k=1}^{\infty} \frac{x^{k}}{k \times k !}, & \text { for } x>0\end{cases} \\
& \psi(x)=-\gamma-\frac{1}{x}+x \sum_{k=1}^{\infty} \frac{1}{k(x+k)}, \text { for } x>0,
\end{aligned}
$$

where $\psi(x)$ is the di-gamma function given by $\psi(x)=d\{\ln [\Gamma(x)]\} / d x$, for $\operatorname{Re}(z)>1$ and $q \neq 0,-1,-2, \ldots$

$$
\zeta(z, q)=\sum_{n=0}^{\infty} \frac{1}{(q+n)^{z}}
$$

and for $\operatorname{Re}(z)<0$ and $0<q \leq 1$,

$$
\zeta(z, q)=\frac{2 \Gamma(1-z)}{(2 \pi)^{1-z}}\left[\sin \left(\frac{z \pi}{2}\right) \sum_{n=0}^{\infty} \frac{\cos (2 \pi q n)}{n^{1-z}}+\cos \left(\frac{z \pi}{2}\right) \sum_{n=0}^{\infty} \frac{\sin (2 \pi q n)}{n^{1-z}}\right]
$$

in which $\gamma \approx 0.577215$ is the Euler's constant.

\section{Definition and Properties}

Here we present the definition of the modified Kies distribution and discuss some of its important properties.

Definition 2.1. A random variable $X$ is said to have a modified Kies distribution with parameters $\alpha, \lambda, \beta, \delta \in R^{+}=(0, \infty)$, written as " $M K D(\alpha, \lambda, \beta, \delta)$ " if its cdf $F(x)$ is of the following form. For $x \in(0, \alpha)$,

$$
F(x)=\left\{1-\exp \left[-\lambda(x /(\alpha-x))^{\beta}\right]\right\}^{\delta} .
$$

On differentiating (2.1) with respect to $\mathrm{x}$, we get the pdf $f(x)$ of the $M K D(\alpha, \lambda, \beta, \delta)$ as in the following, for $x \in(0, \alpha)$.

$$
f(x)=\frac{\alpha \lambda \beta \delta x^{\beta-1} \exp \left[-\lambda(x /(\alpha-x))^{\beta}\right]\left\{1-\exp \left[-\lambda(x /(\alpha-x))^{\beta}\right]\right\}^{\delta-1}}{(\alpha-x)^{\beta+1}}
$$


Clearly when $\delta=1$, the $M K D(\alpha, \lambda, \beta, \delta)$ reduces to the $K D(\alpha, \lambda, \beta)$ with pdf as given in (1.1). When $\alpha=\delta=1$, the $M K D(\alpha, \lambda, \beta, \delta)$ reduces to the exponentiated reduced Kies distribution of Kumar and Dharmaja (2016). Now, we have the following results.

Result 2.1. For any $\alpha, \lambda, \beta$ and $\delta \in R^{+}$, if $X$ follows the $M K D(\alpha, \lambda, \beta, \delta)$, then

(i) $Z_{1}=(X /(\alpha-X))^{\beta}$ follows the generalized exponential distribution of Gupta et al. (1998), with cdf $F_{1}(z)=\{1-\exp (-\lambda z)\}^{\delta}$.

(ii) $Z_{2}=X /(\alpha-X)$ follows the exponentiated Weibull distribution $[E W D(\lambda, \beta, \delta)]$ of Mudholkar and Srivastava (1993) with cdf $F_{2}(z)=\left\{1-\exp \left(-\lambda z^{\beta}\right)\right\}^{\delta}$, which reduces to the Weibull distribution $W D(\lambda, \beta)$, when $\delta=1$.

PROOF. Proof is straight forward and hence omitted

Result 2.2. The survival function $S(x)$ and the hazard function $h(x)$ of the $M K D(\alpha, \lambda, \beta, \delta)$ are respectively,

$$
S(x)=1-F(x) \text { and } h(x)=f(x) / S(x)
$$

for any $x \in(0, \alpha)$ and $\alpha, \lambda, \beta, \delta \in R^{+}$.

Proof. Proof follows from the definition of survival function, hazard rate function and Definition 2.1.

Result 2.3. The hazard function $\mathrm{h}(x)$ of the $M K D(\alpha, \lambda, \beta, \delta)$ is

(i) a bathtub-shaped function in the sense that it is a decreasing function of $x$ for $x<x_{0}$ and an increasing function of $x$ for $x>x_{0}$, in which $x_{0}$ is the solution of the equation

$$
\begin{aligned}
& ((\beta-1) \alpha+2 x)\left\{(F(x))^{(1 / \delta)}-(F(x))^{(\delta+1) / \delta}\right\} \\
& -\alpha \beta \lambda(x /(\alpha-x))^{\beta}\left\{1-\delta \exp \left[-\lambda(x /(\alpha-x))^{\beta}\right]-F(x)\right\}=0,
\end{aligned}
$$

when $(a) \beta<1, \delta \leq 1(b) \beta<1, \delta \geq 1(c) \beta \geq 1, \delta<1$

(ii) an increasing function of $x$ for $\beta \geq 1, \delta \geq 1$

Proof. Proof follows by taking the derivative of the hazard rate function with respect to $x$ and on simplification we get,

$$
\begin{aligned}
\frac{h^{\prime}(x)}{h(x)} & =\frac{\left\{1-\exp \left[-(x /(\alpha-x))^{\beta}\right]\right\}^{-1}}{x(b-x)\left(1-\left\{1-\exp \left[-(x /(\alpha-x))^{\beta}\right]\right\}^{\delta}\right)} \\
& \times\left([(\beta-1) \alpha+2 x]\left\{(F(x))^{\frac{1}{\delta}}-(F(x))^{\frac{(\delta+1)}{\delta}}\right\}\right. \\
& \left.-\alpha \beta \lambda\left(\frac{x}{\alpha-x}\right)^{\beta}\left\{1-\delta \exp \left[-\lambda\left(\frac{x}{\alpha-x}\right)^{\beta}\right]-F(x)\right\}\right),
\end{aligned}
$$



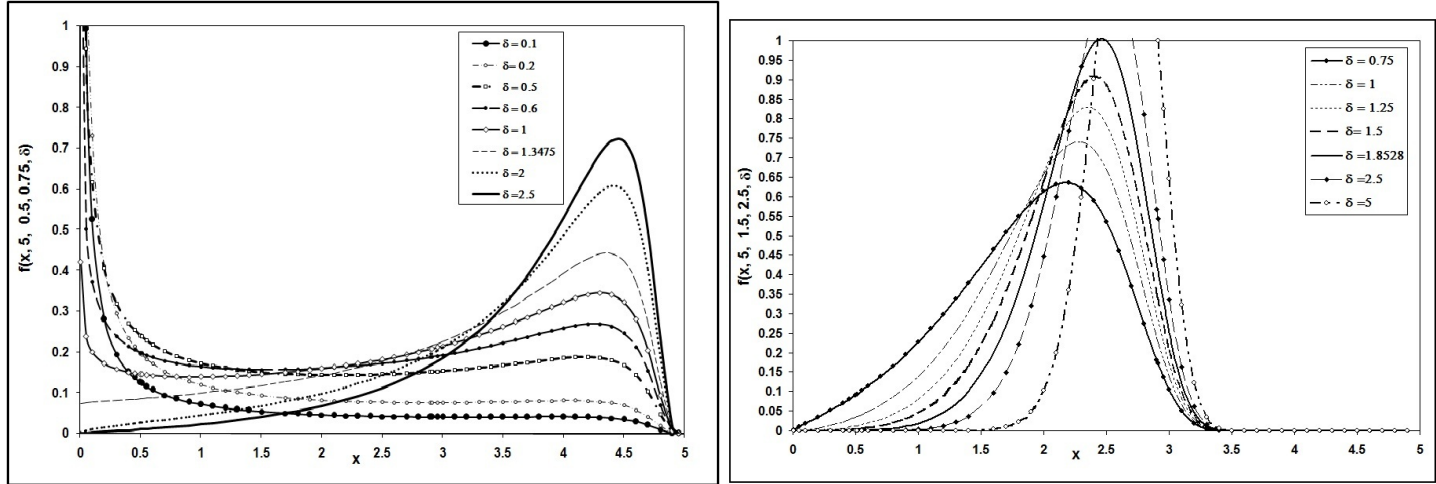

Figure 1: The probability density function plots of the $M K D(5,0.5,0.75, \delta)$ (Left) and $M K D(5,1.5,2.5, \delta)$ (Right).

which leads to the result.

For graphical illustration, we have plotted the pdf $f(x)$ of the $M K D(5,0.5,0.75, \delta)$ and the $\operatorname{MKD}(5,1.5,2.5, \delta)$ for particular choices of $\delta$ in Figure 1, and the hazard rate function $h(x)$ of the $M K D(5,0.5,0.75, \delta)$ and the $M K D(5,1.5,2.5, \delta)$ for particular choices of $\delta$ in Figure 2.

Now we obtain certain percentile measures of the $M K D(\alpha, \lambda, \beta, \delta)$ through the following results.

Result 2.4. For any $\alpha, \lambda, \beta, \delta \in R^{+}$and for any $x_{P} \in(0, \alpha)$ such that $P=F\left(x_{P}\right)$, the percentile function $x_{P}$ of the $M K D(\alpha, \lambda, \beta, \delta)$ with cdf (2.1) is $x_{P}=\alpha \eta_{P}\left(1+\eta_{P}\right)^{-1}$, in which

$$
\eta_{a}=\left[-(1 / \lambda) \ln \left(1-a^{\frac{1}{\delta}}\right)\right]^{\frac{1}{\beta}}
$$

for any $a \in(0,1)$.

Proof. Proof follows by inverting the cdf $F\left(x_{P}\right)=P$ of $M K D(\alpha, \lambda, \beta, \delta)$.

Next we obtain the expression for $r^{\text {th }}$ raw moment of the $M K D(\alpha, \lambda, \beta, \delta)$ through the following result.

Result 2.5. If $X$ follows the $M K D(\alpha, \lambda, \beta, \delta)$ with cdf (2.1), then the $r^{\text {th }}$ raw moment $\mu_{r}^{\prime}$ of the $M K D(\alpha, \lambda, \beta, \delta)$ is the following, in which $\gamma(a, u)$ is as given in (1.3) and for any $i \geq 0, j \geq 0$,

$$
\begin{aligned}
\Lambda_{j}^{(1)}(k-i, \lambda, \beta) & =\frac{\gamma\left(\frac{r+k-j+\beta}{\beta}, \lambda(j+1)\right)}{\lambda^{\frac{r+k-j}{\beta}}(j+1)^{\frac{r+k-j+\beta}{\beta}}} \\
\Lambda_{j}^{(2)}(k-i, \lambda, \beta) & =\lambda^{\frac{k-i}{2 \beta}}(j+1)^{\frac{k-i-2 \beta}{2 \beta}} \exp \left[-\frac{\lambda(j+1)}{2}\right] W_{-\frac{k-i}{2 \beta}, \frac{\beta-(k-i)}{2 \beta}}(\lambda(j+1)),
\end{aligned}
$$

where $W_{k_{1}, k_{2}}(x)$ is the Whittaker function as defined in (1.5). For any $\delta \in N$,

$$
\mu_{r}^{\prime}=\delta \alpha^{r} \sum_{k=0}^{\infty} \sum_{j=0}^{\delta-1} \frac{(-1)^{j+k}{ }^{(\delta-1)} C j}{k !}\left[\Lambda_{j}^{(1)}(k, \lambda, \beta)+\Lambda_{j}^{(2)}(k, \lambda, \beta)\right]
$$



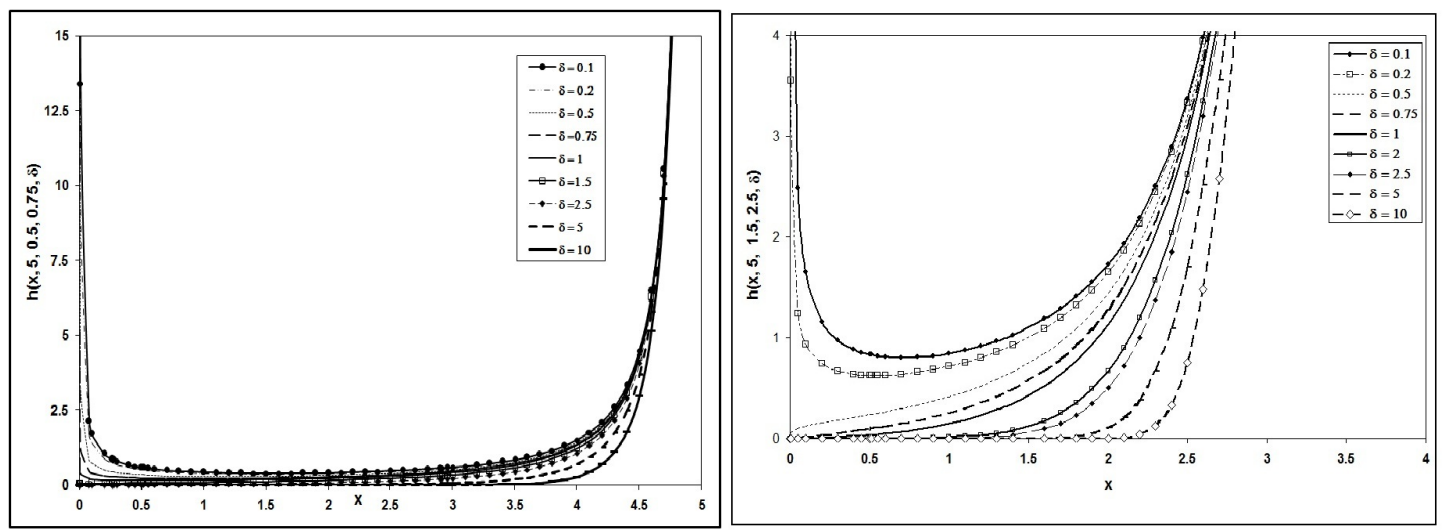

Figure 2: The hazard rate function plots of the $M K D(5,0.5,0.75, \delta)$ (Left) and $M K D(5,1.5,2.5, \delta)$ (Right).

and for $\delta \in R^{+}-N$

$$
\mu_{r}^{\prime}=\delta \alpha^{r} \sum_{k=0}^{\infty} \sum_{j=0}^{k} \frac{(-1)^{j+k}{ }^{(\delta-1)} C j}{k !}\left[\Lambda_{j}^{(1)}(k-j, \lambda, \beta)+\Lambda_{j}^{(2)}(k-j, \lambda, \beta)\right]
$$

PROOF. By definition, the $r^{\text {th }}$ raw moment of $M K D(\alpha, \lambda, \beta, \delta)$ with pdf (2.2) is

$$
\mu_{r}^{\prime}=\int_{0}^{\alpha} \frac{x^{r} \alpha \lambda \beta \delta x^{\beta-1}}{(\alpha-x)^{\beta+1}} \exp \left[-\lambda\left(\frac{x}{\alpha-x}\right)^{\beta}\right]\left\{1-\exp \left[-\lambda\left(\frac{x}{\alpha-x}\right)^{\beta}\right]\right\}^{\delta-1} d x
$$

On substituting $u=\left(\frac{x}{\alpha-x}\right)^{\beta}$ in (2.9), we get

$$
\mu_{r}^{\prime}=\alpha^{r} \lambda \delta \int_{0}^{\infty}\left\{\left(\frac{u^{\frac{1}{\beta}}}{1+u^{\frac{1}{\beta}}}\right)^{r} \exp (-\lambda u)[1-\exp (-\lambda u)]^{\delta-1}\right\} d u
$$

Now we have the following cases.

Case (i) For $\delta \in N$, expanding $\{1-\exp (-\lambda u)\}^{\delta-1}$, we get the following from (2.10).

$$
\mu_{r}^{\prime}=\alpha^{r} \lambda \delta \sum_{j=0}^{\delta-1}\left\{(-1)^{j}{ }^{(\delta-1)} C_{j} \int_{0}^{\infty} \frac{u^{\frac{r}{\beta}}}{\left(1+u^{\frac{1}{\beta}}\right)^{r}} \exp [-\lambda(j+1) u] d u\right\}
$$

On splitting the integral and expanding $\left(1+u^{\frac{1}{\beta}}\right)^{-r}$, we get the following. 


$$
\begin{aligned}
\mu_{r}^{\prime}= & \alpha^{r} \lambda \delta \sum_{k=0}^{\infty} \sum_{j=0}^{\delta-1}(-1)^{j+k}{ }^{(\delta-1)} C_{j} \frac{(r)_{k}}{k !}\left\{\int_{0}^{1} u^{\frac{r+k}{\beta}} \exp [-\lambda(j+1) u] d u\right\} \\
& +\alpha^{r} \lambda \delta \sum_{k=0}^{\infty} \sum_{j=0}^{\delta-1}\left\{(-1)^{j+k}{ }^{(\delta-1)} C_{j} \frac{(r)_{k}}{k !}\left\{\int_{1}^{\infty} \frac{\exp [-\lambda(j+1) u]}{u^{\frac{k}{\beta}}} d u\right\}\right.
\end{aligned}
$$

On substituting $\lambda(j+1) u=v$ in (2.11), we get

$$
\begin{aligned}
\mu_{r}^{\prime} & =\alpha^{r} \lambda \delta \sum_{k=0}^{\infty} \sum_{j=0}^{\delta-1}\left\{(-1)^{j+k} \frac{{ }^{(\delta-1)} C_{j}(r)_{k}}{k !}\left\{\int_{0}^{\lambda(j+1)} \frac{v^{\frac{r+k}{\beta}} \exp (-v)}{\lambda^{\frac{r+k}{\beta}}(j+1)^{\frac{r+k+\beta}{\beta}}} d v\right\}\right\} \\
& +\alpha^{r} \lambda \delta \sum_{k=0}^{\infty} \sum_{j=0}^{\delta-1}\left\{(-1)^{j+k} \frac{{ }^{(\delta-1)} C_{j}(r)_{k}}{k !} \int_{\lambda(j+1)}^{\infty} \frac{\lambda^{\frac{k}{\beta}}(j+1)^{\frac{k}{\beta}-1} \exp (-v)}{v^{\frac{k}{\beta}}} d v\right\},
\end{aligned}
$$

which leads to (2.7) in the light of (1.2), (1.4), (2.5) and (2.6).

Case (ii) For $\delta \in R^{+}-N$, on expanding $\left\{1-\exp (-u)^{\delta-1}\right\}$ in (2.11),

$$
\mu_{r}^{\prime}=\alpha^{r} \lambda \delta \sum_{j=0}^{\infty}\left\{(-1)^{j} \frac{(\delta-1)_{j}}{j !} \int_{0}^{\infty} \frac{u^{\frac{r}{\beta}}}{\left(1+u^{\frac{1}{\beta}}\right)^{r}} \exp [-\lambda(j+1) u] d u\right\}
$$

On splitting the integral and expanding $\left(1+u^{\frac{1}{\beta}}\right)^{-r}$ in (2.13) to get the following.

$$
\begin{array}{r}
\mu_{r}^{\prime}=\alpha^{r} \delta \sum_{k=0}^{\infty} \sum_{j=0}^{\infty}\left\{(-1)^{j+k} \frac{(\delta-1)_{j}(r)_{k}}{j ! k !}\left\{\int_{0}^{1} u^{\frac{r+k}{\beta}} \exp [-\lambda(j+1) u] d u\right\}\right\} \\
+\alpha^{r} \delta \sum_{k=0}^{\infty} \sum_{j=0}^{\infty}\left\{(-1)^{j+k} \frac{(\delta-1)_{j}(r)_{k}}{j ! k !}\left\{\int_{1}^{\infty} \frac{\exp [-\lambda(j+1) u]}{u^{\frac{k}{\beta}}} d u\right\}\right\}
\end{array}
$$

On substituting $\lambda(j+1) u=v$ in (2.14), 

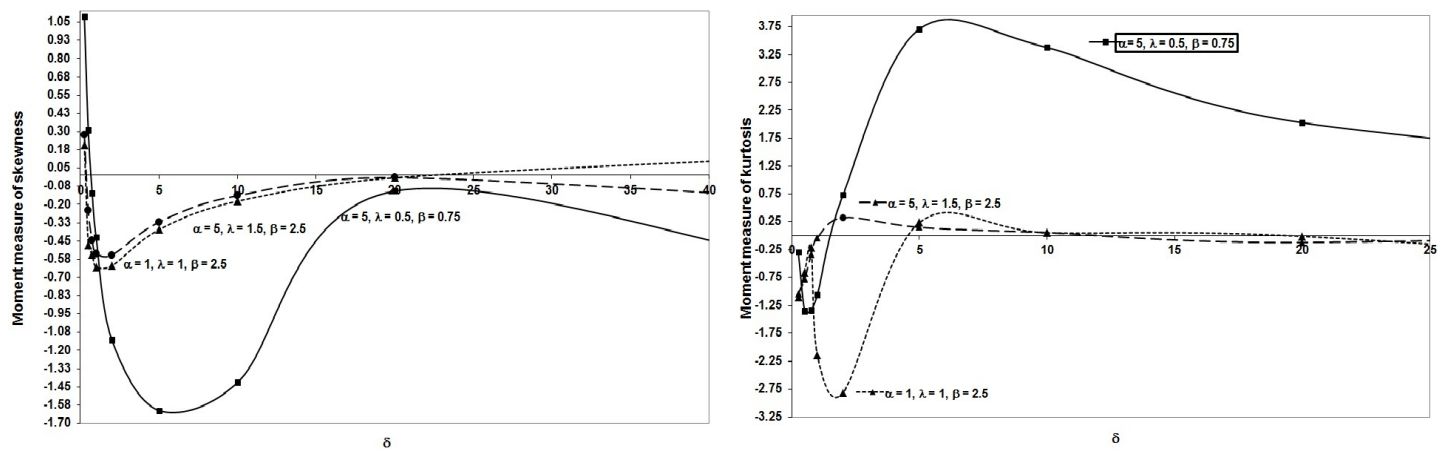

Figure 3: The plots of moment measures of skewness (Left) and kurtosis (Right) of $M K D(\alpha, \lambda, \beta, \delta)$ for particular values of the parameters.

$$
\begin{aligned}
& \mu_{r}^{\prime}=\alpha^{r} \delta \sum_{k=0}^{\infty} \sum_{j=0}^{\infty}\left\{(-1)^{j+k} \frac{(\delta-1)_{j}(r)_{k}}{j ! k !}\left\{\int_{0}^{\lambda(j+1)} \frac{v^{\frac{r+k}{\beta}} \exp (-v)}{\lambda^{\frac{r+k}{\beta}}(j+1)^{\frac{r+k+\beta}{\beta}}} d v\right\}\right\} \\
&+\alpha^{r} \delta \sum_{k=0}^{\infty} \sum_{j=0}^{\infty}\left\{(-1)^{j+k} \frac{(\delta-1)_{j}(r)_{k}}{j ! k !}\left\{\int_{\lambda(j+1)}^{\infty} \frac{\lambda^{\frac{k}{\beta}} \exp (-v)}{(j+1)^{\frac{(\beta-k)}{\beta}}} v^{\frac{k}{\beta}} d v\right\}\right\} \\
& \mu_{r}^{\prime}=\alpha^{r} \delta \sum_{k=0}^{\infty} \sum_{j=0}^{\infty}\left\{(-1)^{j+k} \frac{(\delta-1)_{j}(r)_{k}}{j ! k !}\left[\Lambda_{j}^{(1)}(k, \lambda, \beta)+\Lambda_{j}^{(2)}(k, \lambda, \beta)\right]\right\}
\end{aligned}
$$

which leads to (2.8) in the light of (1.2), (1.4), (1.6), (2.5) and (2.6).

By using Result 2.5, we have computed the values of the moment measure of skewness $\gamma_{1}(=$ $\mu_{3} / \mu_{2}^{3 / 2}$, in which $\mu_{3}$ is the third central moment) and the moment measure of kurtosis $\gamma_{2}(=$ $\left(\mu_{4} / \mu_{2}^{2}\right)-3$, in which $\mu_{4}$ is the fourth central moment) for particular values of its parameters and obtained the graphs in Figure 3.

Result 2.6. Let $X$ be the strength of a system which is subjected to a stress $Y$, and if $X$ follows $M K D\left(\alpha, \lambda, \beta, \delta_{1}\right)$ and $Y$ follows $M K D\left(\alpha, \lambda, \beta, \delta_{2}\right)$, provided $X$ and $Y$ are statistically independent random variables, then $R=P(Y<X)$, the measure of system performance (stress-strength reliability measure) is

$$
R=\delta_{1} /\left(\delta_{1}+\delta_{2}\right)
$$

Proof. Let $f_{1}(x)$ denote the pdf of $X$ and $f_{2}(x)$ denote the pdf of $Y$, then

$$
P(Y<X)=\int_{0}^{\alpha}\left[\left(\int_{0}^{x} f_{2}(y) d y\right) f_{1}(x)\right] d x
$$


By using (2.1), we obtain the following from (2.16).

$$
P(Y<X)=\int_{0}^{\alpha} \frac{\alpha \beta \delta_{1} x^{\beta-1}\left\{1-\exp \left[-\lambda(x /(\alpha-x))^{\beta}\right]\right\}^{\delta_{1}+\delta_{2}-1}}{(\alpha-x)^{\beta+1} \exp \left[\lambda(x /(\alpha-x))^{\beta}\right]} d x
$$

On substituting $u=1-\exp \left[-\lambda(x /(\alpha-x))^{\beta}\right]$ in (2.17), we get $P(Y<x)=\delta_{1} \int_{0}^{1} u^{\delta_{1}+\delta_{2}-1} d u$ which gives (2.15).

Analogous to certain characteristic property enjoyed by Weibull distribution, we obtain similar characteristic property of $M K D(\alpha, \lambda, \beta, \delta)$ through the following theorems.

Theorem 1. If $X$ follows $M K D(\alpha, \lambda, \beta, \delta)$ with $c d f F(x)$ as given in (2.1), then for any $y \in[0, \alpha)$, and for every $0 \leq x \leq \alpha<\infty, \lambda>0, \beta>0$, and $\delta>0$,

$$
\begin{aligned}
& \text { (i) } E\{(1-F(x)) \mid X>y\}=(1-F(x)) / 2, \\
& \text { (ii) } E\{\ln (1-F(x)) \mid X>y\}=\ln [1-F(x)]-1 \\
& \text { (iii) } E\left\{\delta^{-1} \ln [1-F(x)] \mid X \leq y\right\}=\delta^{-1} \ln [1-F(x)]-\delta^{-1} .
\end{aligned}
$$

PROOF. Since the cdf $F(x)$ of $M K D(\alpha, \lambda, \beta, \delta)$ given in (2.1) has the form

$$
F(x)= \begin{cases}0 & \text { for } x<0 \\ \left\{1-\exp \left[-\lambda(x /(\alpha-x))^{\beta}\right]\right\}^{\delta} & \text { for } x \in[0, \alpha) \\ 1 & \text { for } x \geq \alpha\end{cases}
$$

and $\phi_{1}(x)=1-F(x)$ is real valued, continuous and differentiable function on $[0, \alpha)$ with $E\left[\phi_{1}(X)\right]=1 / 2, g(k)=0, \psi(k)=1 / 2$ and by Theorem 7 on Page 260 of Rinne (2009), we obtain (2.17).

Since the cdf $F(x)$ of the the $M K D(\alpha, \lambda, \beta, \delta)$ given in (2.1) has the form

$$
F(x)= \begin{cases}0 & \text { for } x<0 \\ 1-\exp \left[\ln \left(1-\left\{1-\exp \left[-\lambda(x /(\alpha-x))^{\beta}\right]\right\}^{\delta}\right)\right] & \text { for } x \in[0, \alpha) \\ 1 & \text { for } x \geq \alpha\end{cases}
$$

and $\phi_{2}(x)=\ln (1-F(x))$ is real valued, continuous and differentiable function on $(0, \alpha)$ with $\lim _{x \uparrow \alpha} \phi_{2}(x)=-\infty$ and $d=-1$, by Theorem 8 on Page 262 of Rinne (2009), we obtain (2.18).

Further, since the cdf $F(x)$ of the $M K D(\alpha, \lambda, \beta, \delta)$ given in (2.1) takes the form

$$
F(x)= \begin{cases}0 & \text { for } x<0 \\ \exp \left[\delta \ln \left\{1-\exp \left[-\lambda(x /(\alpha-x))^{\beta}\right]\right\}\right] & \text { for } x \in[0, \alpha) \\ 1 & \text { for } x \geq \alpha\end{cases}
$$


and $\phi_{3}(x)=(1 / \delta) \ln [F(x)]$ for $0 \leq x \leq \alpha<\infty$ is a real-valued monotone function continuously differentiable on $(0, \alpha]$ with $\lim _{x \downarrow a} \phi_{3}(x)=-\infty$ with $E\left[\phi_{3}(X)\right]=-1 / \delta, d=-1 / \delta$ and by Theorem 9 on Page 264 of Rinne (2009), we obtain (2.19).

Theorem 2. Let $X_{1}, \ldots, X_{n}$ be $n$ independent and identically distributed (i.i.d.) random variables following the $\operatorname{MKD}(\alpha, \lambda, \beta, \delta)$ with $c d f$ (2.1) and let $Y=\max \left(X_{1}, \ldots, X_{n}\right)$. Then $Y$ follows the $M K D(\alpha, \lambda, \beta, n \delta)$. Conversely, if $Y$ follows the $M K D(a, b, \eta, \omega)$, then each $X_{i}$ follows $M K D\left(a, b, \eta, \omega n^{-1}\right), i=1,2, \ldots, n$.

Proof. If $X_{1}, \ldots, X_{n}$ are i.i.d. $M K D(\alpha, \lambda, \beta, \delta)$ variates each with pdf (2.2), the pdf $f_{n}(y)$ of $Y=\max \left(X_{1}, \ldots, X_{n}\right)$ is the following, for any $\alpha>0, \lambda>0, \delta>0$ and $\beta>0$.

$$
f_{n}(y)=\frac{\alpha \lambda \beta n \delta y^{\beta-1}}{(\alpha-y)^{\beta+1}} \exp \left[-\lambda(y /(\alpha-y))^{\beta}\right]\left\{1-\exp \left[-\lambda(y /(\alpha-y))^{\beta}\right]\right\}^{n \delta-1}
$$

Since the pdf $g_{n}(z)$ of maximum of $n$ i.i.d. random variates each with pdf $g(z)$ and $\operatorname{cdf} G(z)$ is $g_{n}(z)=n g(z)[G(z)]^{n-1}$. Thus $Y$ follows the $M K D(\alpha, \lambda, \beta, n \delta)$.

Conversely, assume that $Y=\max \left(X_{1}, \ldots, X_{n}\right)$ follows the $M K D(a, b, \eta, \omega)$, then the cdf $F_{n}(y)$ of $Y$ is

$$
F_{n}(y)=\left\{1-\exp \left[-b(y /(a-y))^{\eta}\right]\right\}^{\omega}
$$

in the light of (2.1). For any i.i.d. random variates $Z_{1}, \ldots, Z_{n}$ each with $\operatorname{cdf} G(z)$, the $\operatorname{cdf} G_{n}(z)$ of $Z=\max \left(Z_{1}, \ldots, Z_{n}\right)$ is

$$
G_{n}(z)=[G(z)]^{n} .
$$

Now we obtain the following from (2.20) in the light of (2.21).

$$
[F(y)]^{n}=\left\{1-\exp \left[-b\left(\frac{y}{a-y}\right)^{\eta}\right]\right\}^{\omega} .
$$

Thus the pdf of $X_{1}$ is

$$
f(y)=F^{\prime}(y)=a b \eta \frac{\omega}{n} \frac{y^{\eta-1}}{(a-y)^{\eta+1}} \exp \left[-b\left(\frac{y}{a-y}\right)^{\eta}\right]\left\{1-\exp \left[-b\left(\frac{y}{a-y}\right)^{\eta}\right]\right\}^{\frac{\omega}{n}-1}
$$

\section{Estimation}

Here we discuss the maximum likelihood estimation of the parameters of the $M K D(\alpha, \lambda, \beta, \delta)$. Consider the following log-likelihood function $\ell$ of a random sample $X_{1}, \ldots, X_{n}$ taken from a population following the $M K D(\alpha, \lambda, \beta, \delta)$ with pdf (2.2).

$$
\begin{aligned}
\ell & =n \ln (\delta)+n \ln (\beta)+n \ln (\lambda)+n \ln (\alpha)+(\beta-1) \sum_{i=1}^{n} \ln \left(x_{i}\right)-\lambda \sum_{i=1}^{n}\left(\frac{x_{i}}{\alpha-x_{i}}\right)^{\beta} \\
& -(\beta+1) \sum_{i=1}^{n} \ln \left(\alpha-x_{i}\right)+(\delta-1) \sum_{i=1}^{n} \ln \left\{1-\exp \left[-\lambda\left(\frac{x}{\alpha-x}\right)^{\beta}\right]\right\}
\end{aligned}
$$


On differentiating the log-likelihood function (3.1) with respect to the respective parameters and equating to zero, we can obtain the likelihood equations. On solving these likelihood equations one can obtain the maximum likelihood estimates (MLE)of the parameters of $M K D(\alpha, \lambda, \beta, \delta)$. These likelihood equations do not always have a unique solution because the $M K D(\alpha, \lambda, \beta, \delta)$ is not a regular model. By using the package 'maxLik'-R (cf. Henningsen and Toomet, 2011), we observed that the second order partial derivatives of the log-likelihood function with respect to the parameters gives negative values for $\alpha>0, \lambda>0, \beta>0$ and $\delta>0$. The expressions of elements of the corresponding Fisher information matrix $I(\theta)$ are given in Appendix.

\section{Applications}

In this section we discuss certain applications of the $M K D(\alpha, \lambda, \beta, \delta)$ with the help of the following data sets.

Data set 1 The data set on 30 "times of failure and running times" for a sample of devices from a field-tracking study of a larger system taken from Meekar and Escobar (1998) is:

$275,13,147,23,181,30,65,10,300,173,106,300,300,212,300,300,300,2,261,293$, $88,247,28,143,300,23,300,80,245,266$

Data set 2 The data set on 23 "time between failures of secondary reactor pumps" taken from Bebbington et al. (2007) is:

$2.160,0.150,4.082,0.746,0.358,0.199,0.402,0.101,0.605,0.954,1.359,0.273,0.491$,

$3.465,0.070,6.560,1.060,0.062,4.992,0.614,5.320,0.347,1.921$

Data set 3 This data set is taken from Aarset (1987) which is "on lifetimes of 50 components" and they are given as follows:

$0.1,0.2,1,1,1,1,1,2,3,6,7,11,12,18,18,18,18,18,21,32,36,40,45$,

$46,47,50,55,60,63,63,67,67,67,67,72,75,79,82,82,83,84,84,84,85$,

$85,85,85,85,86,86$

Data set 4 The data set on 19 "initial remission times of leukemia patients" taken from Lee and Wang (2003) is:

$8,10,10,12,14,20,48,70,75,99,103,161,162,169,195,199,217,220,245$

We have fitted the $M K D(\alpha, \lambda, \beta, \delta)$ to the data sets with the help of the package 'maxLik'-R (cf. Henningsen and Toomet, 2011). We have fitted the following models to the four data sets for comparison 
(a) the $K D(\alpha, \lambda, \beta)$, in which $0 \leq x \leq \alpha<\infty, \lambda>0$ and $\beta>0$ (cf. Kumar and Dharmaja, 2014),

(b) the $B G W D(\mu, \theta, \sigma, \tau, \rho)$, in which $x>0, \mu, \theta, \sigma, \tau$ and $\rho>0$. (cf. Singla et al., 2012),

(c) the $B W D(\mu, \theta, \sigma, \rho)$, in which $x>0, \mu, \theta, \sigma$ and $\rho>0$ (cf. Famoye et al., 2005) and

(d) the $E W D(\mu, \sigma, \rho)$, in which $x>0, \mu, \theta, \sigma$ and $\rho>0$. (cf. Mudholkar et al., 1995).

Then we compared the fitted model the $M K D(\alpha, \lambda, \beta, \delta)$ with that of fitted models-the $K D(\alpha, \lambda, \beta)$, the $B G W D(\mu, \theta, \sigma, \rho, \tau)$, the $B W D(\mu, \theta, \sigma, \rho)$ and the $E W D(\mu, \sigma, \rho)$. For model comparison, we have computed the values of log-likelihood, the Akaike information criterion (AIC), Bayesian information criterion (BIC) and the second order Akaike information criterion (AICc) and included in Table 1. It is seen that the values of the AIC, the BIC and the AICc of each data set in the case of the $M K D(\alpha, \lambda, \beta, \delta)$ model is the lowest. Hence the $M K D(\alpha, \lambda, \beta, \delta)$ can be viewed as the best model compared to other existing models considered in this section. Further, we have plotted the cdf of these fitted models against the corresponding empirical distribution in Figure 4. Figure 4 also supports the above conclusion that the $M K D(\alpha, \lambda, \beta, \delta)$ gives a better fit to each data set compared to other generalized Weibull models considered in this paper. Moreover, using these fitted models, we have obtained the Weibull Probability Plots (WPP plots) as in Figure 5 corresponding to the two data sets for comparing the models. These plots also indicate that the $M K D(\alpha, \lambda, \beta, \delta)$ as the best model to the data sets considered in the paper compared to other existing models.

\section{Simulation}

In order to assess the performance of the maximum likelihood estimators of the parameters of the $M K D(\alpha, \lambda, \beta, \delta)$, we have carried out a brief simulation study. We, simulated datasets by adapting probability integral transform method based on the following sets of of parameters according to the nature of the skewness.

(i) $\alpha=5, \lambda=1.5, \beta=2.5$ and $\delta=0.2$ (positively skewed)

(ii) $\alpha=20.28, \lambda=27.13, \beta=10.51$ and $\delta=0.16$ (negatively skewed).

We considered 200 bootstrap samples of sizes $n=10,25,50$ and 100 for comparison and computed average bias and mean squared errors (MSEs) in each case. The results obtained are summarized in Table 2. From Table 2, it can be observed that both the average bias and MSEs of the estimators are in decreasing order, as sample size increases. 


\begin{tabular}{|c|c|c|c|c|c|c|c|c|c|c|}
\hline$\frac{\vartheta}{\varepsilon}$ & $\begin{array}{l}\frac{n}{0} \\
\bar{c} \\
0 \\
0\end{array}$ & 䯋 & 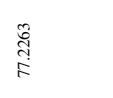 & 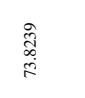 & 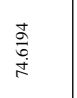 & & 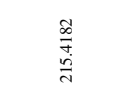 & 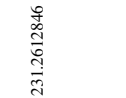 & $\begin{array}{l}\text { के } \\
\text { के } \\
\text { 就 }\end{array}$ & 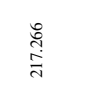 \\
\hline$\frac{\nu}{\mu}$ & $\frac{\substack{0 \\
\frac{\partial}{2}}}{2}$ & $\frac{8}{8}$ & $\begin{array}{l}\frac{7}{2} \\
\frac{2}{2} \\
2\end{array}$ & 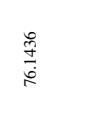 & $\begin{array}{l}0 \\
\substack{0 \\
0 \\
0 \\
\alpha}\end{array}$ & & $\begin{array}{l}\frac{0}{0} \\
\frac{0}{2} \\
\frac{0}{0} \\
\frac{0}{2}\end{array}$ & 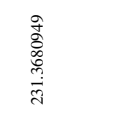 & 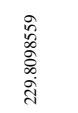 & 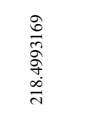 \\
\hline 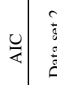 & 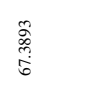 & 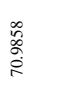 & 总 & 产 & 产 & 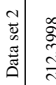 & $\begin{array}{l}\frac{\infty}{\infty} \\
\frac{\infty}{\pi} \\
\frac{\infty}{\pi}\end{array}$ & 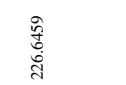 & 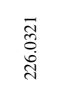 & $\begin{array}{l}\frac{8}{0} \\
\text { ond } \\
\text { on }\end{array}$ \\
\hline |ٕ: & 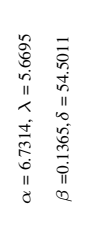 & 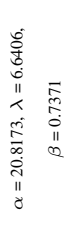 & 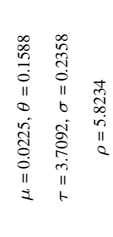 & 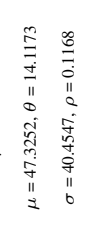 & 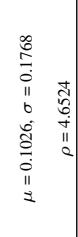 & & 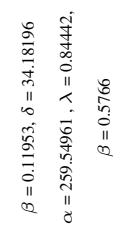 & 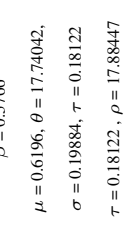 & 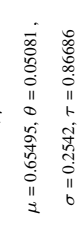 & 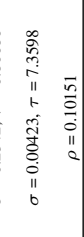 \\
\hline$\stackrel{\bullet}{\&}$ & 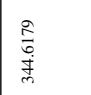 & 离 & 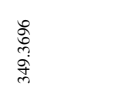 & 密 & $\begin{array}{l}\text { 命 } \\
\text { c్d } \\
\text { d. }\end{array}$ & 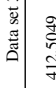 & 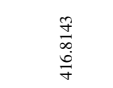 & 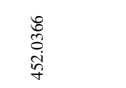 & 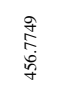 & 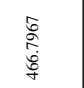 \\
\hline$\frac{u}{m}$ & 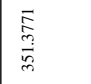 & $\begin{array}{l}\text { 高 } \\
\text { ồ } \\
\text { de }\end{array}$ & $\begin{array}{l}\overline{8} \\
6 \\
6 \\
6\end{array}$ & $\begin{array}{l}\text { 今े } \\
\text { ò } \\
\text { D. }\end{array}$ & 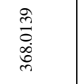 & 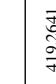 & 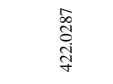 & 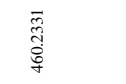 & $\begin{array}{l}\text { 奉 } \\
\text { 索 }\end{array}$ & 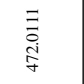 \\
\hline$\frac{u}{4}$ & 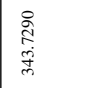 & 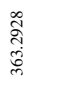 & 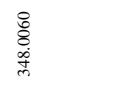 & 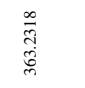 & $\begin{array}{l}\infty \\
\text { ते } \\
\text { d్ల }\end{array}$ & $\frac{8}{0}$ & 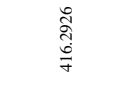 & 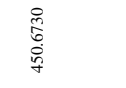 & 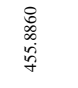 & 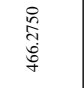 \\
\hline |ٕ: & 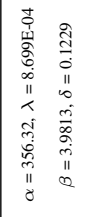 & 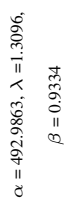 & 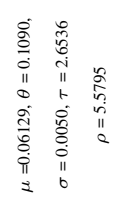 & 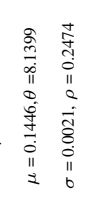 & 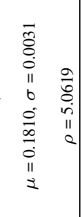 & & 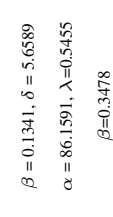 & 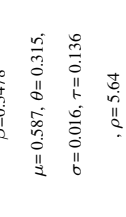 & 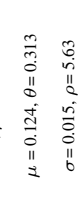 & 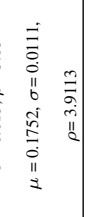 \\
\hline$\frac{\bar{a}}{\frac{\mathrm{s}}{2}}$ & 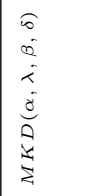 & 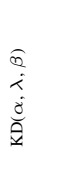 & 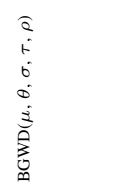 & 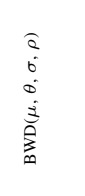 & 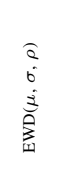 & 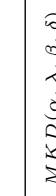 & 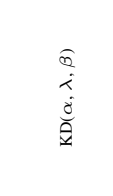 & 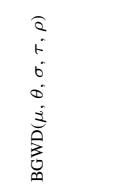 & $\begin{array}{l}\hat{a} \\
0 \\
0 \\
\dot{0} \\
\dot{0} \\
\hat{\bar{y}}\end{array}$ & 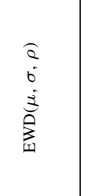 \\
\hline
\end{tabular}


Table 2: Average bias and mean squared errors (in brackets) of the MLEs of the $\operatorname{GKD}(\alpha, \lambda, \beta, \rho)$ based on simulated data sets for the parameter sets (i) $\alpha=5, \lambda=1.5, \beta=2.5, \rho=0.2$ and (ii) $\alpha=20.28, \lambda=27.13, \beta=10.51, \rho=0.16$.

\begin{tabular}{|c|c|c|c|c|c|}
\hline & \multirow[b]{2}{*}{ Parameter } & \multicolumn{4}{|c|}{ Sample size } \\
\hline & & 10 & 25 & 50 & 100 \\
\hline Parameter & $\alpha$ & -0.8596 & 0.1329 & -0.1162 & -0.0939 \\
\hline \multirow[t]{7}{*}{ Set (i) } & & $(1.1689)$ & $(1.1459)$ & $(0.2776)$ & $(0.0308)$ \\
\hline & $\lambda$ & -0.8880 & 0.3561 & -0.2148 & 0.0072 \\
\hline & & (3.0252) & $(2.3675)$ & $(0.2435)$ & $(0.0563)$ \\
\hline & $\beta$ & 1.8214 & -0.1367 & -0.0718 & 0.0653 \\
\hline & & $(4.4569)$ & $(1.2201)$ & $(0.0668)$ & $(0.0286)$ \\
\hline & $\rho$ & 0.2563 & 0.1565 & 0.0597 & 0.0287 \\
\hline & & $(2.9952)$ & $(0.1266)$ & $(0.0047)$ & $(0.0011)$ \\
\hline Parameter & $\alpha$ & -2.6349 & -0.2522 & -0.1579 & 0.1011 \\
\hline \multirow[t]{7}{*}{ Set (ii) } & & (7.9427) & $(0.0538)$ & $(0.0368)$ & $(0.0112)$ \\
\hline & $\lambda$ & -0.1548 & -0.0677 & 0.0488 & 0.0226 \\
\hline & & $(0.0439)$ & $(0.0068)$ & $(0.0054)$ & $(7.90 \mathrm{E}-04)$ \\
\hline & $\beta$ & 7.2212 & 0.6093 & -0.1937 & -0.1008 \\
\hline & & $(68.3939)$ & $(0.3713)$ & $(0.0379)$ & $(0.0125)$ \\
\hline & $\rho$ & -0.0833 & -0.0490 & 0.0107 & 0.0053 \\
\hline & & $(0.0069)$ & $(0.0024)$ & (8.00E-04) & $(2.89 \mathrm{E}-05)$ \\
\hline
\end{tabular}

\section{Conclusion}

In this paper, an exponentiated version of the Kies distribution namely "the modified Kies distribution $M K D(\alpha, \lambda, \beta, \delta)$ " is introduced as a generalization of the exponentiated reduced Kies distribution $\operatorname{ERKD}(\beta, \delta)$ of Kumar and Dharmaja (2016) and investigated several properties of the distribution. It can be noted that the support of the ERKD is over the range $(0,1)$ while that of the $M K D(\alpha, \lambda, \beta, \delta)$, is over the range $(0, \alpha)$, for $\alpha>0$. So, in certain practical situations, the data need to be transformed while fitting the $\operatorname{ERKD}(\beta, \delta)$, and this drawback is investigated in the case of $M K D(\alpha, \lambda, \beta, \delta)$. Further the inclusion of scale parameter $\lambda$ helps to create more flexibility in practical point of view and thus the practical relevance of the model is quite obvious from the fitting of the model to various data sets considered in Section 4 of the paper. Further a brief simulation study is carried out for examining the asymptotic behavior of the maximum likelihood estimates of the parameters of the distribution.

\section{Acknowledgements}

The authors would like to express their sincere gratitude to the Editor, the Associate Editor and the anonymous Referee for their valuable comments on an earlier version of the paper, which greatly improved the quality presentation of the paper. 

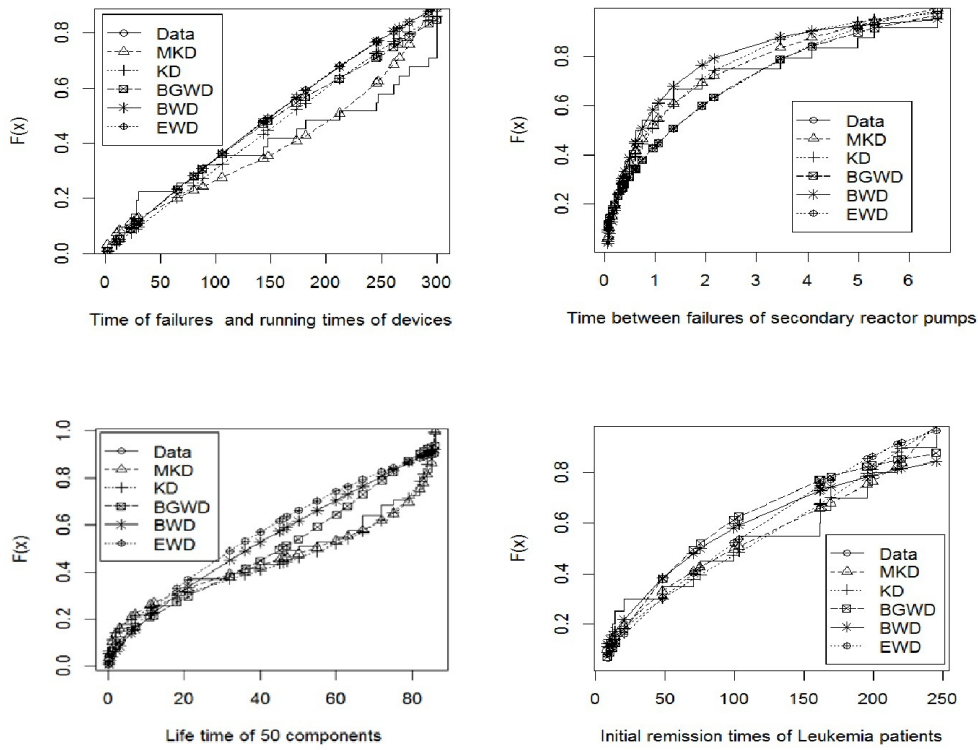

Figure 4: Empirical and Fitted distribution function plots for the Data set 1 ( Top Left), Data set 2 (Top Right) and Data set 3 (Bottom Left) and Data set 4 (Bottom Right).

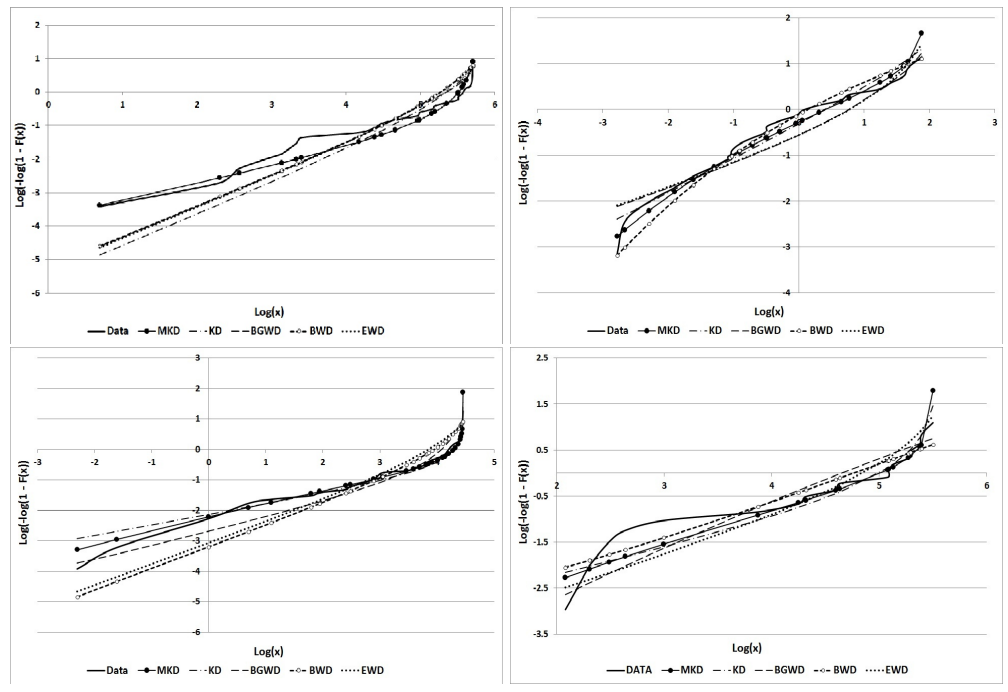

Figure 5: Weibull Probability Plots for Data set 1 ( Top Left ), Data set 2 (Top Right), Data set 3 (Bottom Left) and Data set 4 (Bottom Right). 


\section{A Elements of Information Matrix}

$$
\begin{aligned}
& I_{11}=-\frac{1}{\alpha^{2}}+\frac{\lambda \delta(\beta+1)}{\alpha^{2}} \sum_{j=0}^{\delta-1}(-1)^{j(\delta-1)} C_{j}\left\{\frac{1}{\lambda(j+1)}+\frac{2 \Gamma\left(\frac{1}{\beta}+1\right)}{[\lambda(j+1)]^{\frac{1}{\beta}+1}}+\frac{\Gamma\left(\frac{2}{\beta}+1\right)}{[\lambda(j+1)]^{\frac{2}{\beta}+1}}\right\} \\
& -\frac{\lambda^{2} \beta \delta(\beta+1)}{\alpha^{2}} \sum_{j=0}^{\delta-1}(-1)^{j}{ }^{(\delta-1)} C_{j}\left\{\frac{1}{[\lambda(j+2)]^{2}}+\frac{2 \Gamma\left(\frac{1}{\beta}+1\right)}{[\lambda(j+2)]^{\frac{1}{\beta}+1}}+\frac{\Gamma\left(\frac{2}{\beta}+1\right)}{[\lambda(j+2)]^{\frac{2}{\beta}+1}}\right\} \\
& +\frac{\lambda^{2} \beta \delta(\beta+1)(\delta-1)}{\alpha^{2}} \sum_{j=0}^{\delta-2}(-1)^{j(\delta-2)} C_{j}\left\{\frac{1}{[\lambda(j+2)]^{2}}+\frac{2 \Gamma\left(\frac{1}{\beta}+1\right)}{[\lambda(j+2)]^{\frac{1}{\beta}+1}}+\frac{\Gamma\left(\frac{2}{\beta}+1\right)}{[\lambda(j+2)]^{\frac{2}{\beta}+1}}\right\} \\
& +\frac{\lambda^{3} \beta^{2} \delta(\delta-1)}{\alpha^{2}} \sum_{j=0}^{\delta-3}(-1)^{j}{ }^{(\delta-3)} C_{j}\left\{\frac{2}{[\lambda(j+3)]^{2}}+\frac{2 \Gamma\left(\frac{1}{\beta}+2\right)}{[\lambda(j+3)]^{\frac{1}{\beta}+1}}+\frac{\Gamma\left(\frac{2}{\beta}+2\right)}{[\lambda(j+3)]^{\frac{2}{\beta}+1}}\right\}, \\
& I_{12}=I_{21}=\frac{\lambda \beta \delta}{\alpha} \sum_{j=0}^{\delta-1}(-1)^{j}{ }^{(\delta-1)} C_{j}\left\{\frac{1}{[\lambda(j+1)]^{2}}+\frac{\Gamma\left(\frac{1}{\beta}+2\right)}{[\lambda(j+1)]^{\frac{1}{\beta}+2}}\right\} \\
& -\frac{\lambda \beta \delta(\delta-1)}{\alpha} \sum_{j=0}^{\delta-2}(-1)^{j}{ }^{(\delta-2)} C_{j}\left\{\frac{1}{[\lambda(j+2)]^{2}}+\frac{\Gamma\left(\frac{1}{\beta}+2\right)}{[\lambda(j+2)]^{\frac{1}{\beta}+2}}\right\} \\
& +\frac{\lambda^{2} \beta \delta(\delta-1)}{\alpha} \sum_{j=0}^{\delta-3}(-1)^{j}{ }^{(\delta-3)} C_{j}\left\{\frac{2}{[\lambda(j+2)]^{3}}+\frac{\Gamma\left(\frac{1}{\beta}+3\right)}{[\lambda(j+2)]^{\frac{1}{\beta}+3}}\right\}, \\
& I_{13}=I_{31}=-\frac{\lambda \delta}{\alpha} \sum_{j=0}^{\delta-1}(-1)^{j(\delta-1)} C_{j}\left\{\frac{1}{[\lambda(j+1)]}+\frac{\Gamma\left(\frac{1}{\beta}+1\right)}{[\lambda(j+1)]^{\frac{1}{\beta}+1}}\right\} \\
& +\frac{\lambda \delta}{\alpha} \sum_{j=0}^{\delta-1}(-1)^{j(\delta-1)} C_{j}\left\{\frac{1}{[\lambda(j+1)]^{2}}+\frac{\Gamma\left(\frac{1}{\beta}+2\right)}{[\lambda(j+1)]^{\frac{1}{\beta}+2}}\right\} \\
& +\frac{\lambda \delta}{\alpha} \sum_{j=0}^{\delta-1}(-1)^{j}{ }^{(\delta-1)} C_{j}\left\{\frac{\{\psi(2)-\ln [\lambda(j+1)]\}}{[\lambda(j+1)]}+\frac{\Gamma\left(\frac{1}{\beta}+2\right)\left\{\psi\left(\frac{1}{\beta}+2\right)-\ln [\lambda(j+1)]\right\}}{[\lambda(j+1)]^{\frac{1}{\beta}+2}}\right\} \\
& -\frac{\lambda^{2} \delta(\delta-1)}{\alpha} \sum_{j=0}^{\delta-2}(-1)^{j}{ }^{(\delta-2)} C_{j}\left\{\frac{1}{[\lambda(j+2)]^{2}}+\frac{\Gamma\left(\frac{1}{\beta}+2\right)}{[\lambda(j+2)]^{\frac{1}{\beta}+2}}\right\}, \\
& I_{14}=I_{41}=-\frac{\lambda^{2} \beta \delta}{\alpha} \sum_{j=0}^{\delta-2}(-1)^{j(\delta-2)} C_{j}\left\{\frac{1}{[\lambda(j+2)]^{2}}+\frac{\Gamma\left(\frac{1}{\beta}+2\right)}{[\lambda(j+2)]^{\frac{1}{\beta}+2}}\right\} \text {, } \\
& I_{22}=-\frac{1}{\lambda^{2}}-2 \lambda \delta(\delta-1) \sum_{j=0}^{\delta-3} \frac{(-1)^{j(\delta-3)} C_{j}}{[\lambda(j+2)]^{3}} \text {, }
\end{aligned}
$$




$$
\begin{aligned}
& I_{23}=I_{32}=-\frac{\lambda \delta}{\beta} \sum_{j=0}^{\delta-1}(-1)^{j}{ }^{(\delta-1)} C_{j}\left\{\frac{\psi(2)-\ln [\lambda(j+1)]}{[\lambda(j+1)]^{2}}\right\} \\
& +\frac{\lambda \delta(\delta-1)}{\beta} \sum_{j=0}^{\delta-2}(-1)^{j(\delta-2)} C_{j}\left\{\frac{\psi(2)-\ln [\lambda(j+2)]}{[\lambda(j+2)]^{2}}\right\} \\
& -\frac{2 \lambda^{2} \delta(\delta-1)}{\beta} \sum_{j=0}^{\delta-3}(-1)^{j}{ }^{(\delta-3)} C_{j}\left\{\frac{\psi(3)-\ln [\lambda(j+2)]}{[\lambda(j+2)]^{3}}\right\}, \\
& I_{24}=I_{42}=\lambda \delta \sum_{j=0}^{\delta-2}\left\{\frac{(-1)^{j}{ }^{(\delta-2)} C_{j}}{[\lambda(j+2)]^{2}}\right\} \\
& I_{33}=-\frac{1}{\beta^{2}}-\frac{\lambda^{2} \delta}{\beta^{2}} \sum_{j=0}^{\delta-1}(-1)^{j}{ }^{(\delta-1)} C_{j}\left\{\frac{\{\psi(2)-\ln [\lambda(j+1)]\}^{2}+\zeta(2,2)}{[\lambda(j+1)]^{2}}\right\} \\
& +\frac{\lambda^{2} \delta(\delta-1)}{\beta^{2}} \sum_{j=0}^{\delta-2}(-1)^{j(\delta-2)} C_{j}\left\{\frac{\{\psi(2)-\ln [\lambda(j+2)]\}^{2}+\zeta(2,2)}{[\lambda(j+2)]^{2}}\right\} \\
& -\frac{2 \lambda^{3} \delta(\delta-1)}{\beta^{2}} \sum_{j=0}^{\delta-3}(-1)^{j}{ }^{(\delta-3)} C_{j}\left\{\frac{\{\psi(3)-\ln [\lambda(j+2)]\}^{2}+\zeta(2,3)}{[\lambda(j+2)]^{3}}\right\}, \\
& I_{34}=I_{43}=\frac{\lambda^{2} \delta}{\beta} \sum_{j=0}^{\delta-2}(-1)^{j(\delta-2)} C_{j}\left\{\frac{\psi(2)-\ln [\lambda(j+2)]}{[\lambda(j+2)]^{2}}\right\} \text {, } \\
& I_{44}=I_{44}^{\prime}=-\frac{1}{\delta^{2}}, \\
& I_{11}^{\prime}=-\frac{1}{\alpha^{2}}+\frac{\lambda \delta(\beta+1)}{\alpha^{2}} \sum_{j=0}^{\infty} \frac{(-1)^{j}(\delta-1)_{j}}{j !}\left\{\frac{1}{\lambda(j+1)}+\frac{2 \Gamma\left(\frac{1}{\beta}+1\right)}{[\lambda(j+1)]^{\frac{1}{\beta}+1}}+\frac{\Gamma\left(\frac{2}{\beta}+1\right)}{[\lambda(j+1)]^{\frac{2}{\beta}+1}}\right\} \\
& -\frac{\lambda^{2} \beta \delta(\beta+1)}{\alpha^{2}} \sum_{j=0}^{\infty} \frac{(-1)^{j}(\delta-1)_{j}}{j !}\left\{\frac{1}{[\lambda(j+2)]^{2}}+\frac{2 \Gamma\left(\frac{1}{\beta}+1\right)}{[\lambda(j+2)]^{\frac{1}{\beta}+1}}+\frac{\Gamma\left(\frac{2}{\beta}+1\right)}{[\lambda(j+2)]^{\frac{2}{\beta}+1}}\right\} \\
& +\frac{\lambda^{2} \beta \delta(\beta+1)(\delta-1)}{\alpha^{2}} \sum_{j=0}^{\infty} \frac{(-1)^{j}(\delta-2)_{j}}{j !}\left\{\frac{1}{[\lambda(j+2)]^{2}}+\frac{2 \Gamma\left(\frac{1}{\beta}+1\right)}{[\lambda(j+2)]^{\frac{1}{\beta}+1}}+\frac{\Gamma\left(\frac{2}{\beta}+1\right)}{[\lambda(j+2)]^{\frac{2}{\beta}+1}}\right\} \\
& +\frac{\lambda^{3} \beta^{2} \delta(\delta-1)}{\alpha^{2}} \sum_{j=0}^{\infty} \frac{(-1)^{j}(\delta-3)_{j}}{j !}\left\{\frac{2}{[\lambda(j+3)]^{2}}+\frac{2 \Gamma\left(\frac{1}{\beta}+2\right)}{[\lambda(j+3)]^{\frac{1}{\beta}+1}}+\frac{\Gamma\left(\frac{2}{\beta}+2\right)}{[\lambda(j+3)]^{\frac{2}{\beta}+1}}\right\}, \\
& I_{12}^{\prime}=\frac{\lambda \beta \delta}{\alpha} \sum_{j=0}^{\infty} \frac{(-1)^{j}(\delta-1)_{j}}{j !}\left\{\frac{1}{[\lambda(j+1)]^{2}}+\frac{\Gamma\left(\frac{1}{\beta}+2\right)}{[\lambda(j+1)]^{\frac{1}{\beta}+2}}\right\} \\
& -\frac{\lambda \beta \delta(\delta-1)}{\alpha} \sum_{j=0}^{\infty} \frac{(-1)^{j}(\delta-2)_{j}}{j !}\left\{\frac{1}{[\lambda(j+2)]^{2}}+\frac{\Gamma\left(\frac{1}{\beta}+2\right)}{[\lambda(j+2)]^{\frac{1}{\beta}+2}}\right\} \\
& +\frac{\lambda^{2} \beta \delta(\delta-1)}{\alpha} \sum_{j=0}^{\infty} \frac{(-1)^{j}(\delta-3)_{j}}{j !}\left\{\frac{2}{[\lambda(j+2)]^{3}}+\frac{\Gamma\left(\frac{1}{\beta}+3\right)}{[\lambda(j+2)]^{\frac{1}{\beta}+3}}\right\},
\end{aligned}
$$




$$
\begin{aligned}
& I_{13}^{\prime}=I_{31}^{\prime}=-\frac{\lambda \delta}{\alpha} \sum_{j=0}^{\infty} \frac{(-1)^{j}(\delta-1)_{j}}{j !}\left\{\frac{1}{[\lambda(j+1)]}+\frac{\Gamma\left(\frac{1}{\beta}+1\right)}{[\lambda(j+1)]^{\frac{1}{\beta}+1}}\right\} \\
& +\frac{\lambda \delta}{\alpha} \sum_{j=0}^{\infty} \frac{(-1)^{j}(\delta-1)_{j}}{j !}\left\{\frac{1}{[\lambda(j+1)]^{2}}+\frac{\Gamma\left(\frac{1}{\beta}+2\right)}{[\lambda(j+1)]^{\frac{1}{\beta}+2}}\right\} \\
& +\frac{\lambda \delta}{\alpha} \sum_{j=0}^{\infty} \frac{(-1)^{j}(\delta-1)_{j}}{j !}\left\{\frac{\{\psi(2)-\ln [\lambda(j+1)]\}}{[\lambda(j+1)]}+\frac{\Gamma\left(\frac{1}{\beta}+2\right)\left\{\psi\left(\frac{1}{\beta}+2\right)-\ln [\lambda(j+1)]\right\}}{[\lambda(j+1)]^{\frac{1}{\beta}+2}}\right\} \\
& -\frac{\lambda^{2} \delta(\delta-1)}{\alpha} \sum_{j=0}^{\infty} \frac{(-1)^{j}(\delta-2)_{j}}{j !}\left\{\frac{1}{[\lambda(j+2)]^{2}}+\frac{\Gamma\left(\frac{1}{\beta}+2\right)}{[\lambda(j+2)]^{\frac{1}{\beta}+2}}\right\} \\
& -\frac{\lambda^{2} \delta(\delta-1)}{\alpha} \sum_{j=0}^{\infty} \frac{(-1)^{j}(\delta-2)_{j}}{j !}\left\{\frac{\{\psi(2)-\ln [\lambda(j+2)]\}}{[\lambda(j+2)]^{2}}+\frac{\Gamma\left(\frac{1}{\beta}+3\right)\left\{\psi\left(\frac{1}{\beta}+3\right)-\ln [\lambda(j+2)]\right\}}{[\lambda(j+2)]^{\frac{1}{\beta}+3}}\right\} \\
& +\frac{\lambda^{2} \delta(\delta-1)}{\alpha} \sum_{j=0}^{\infty} \frac{(-1)^{j}(\delta-3)_{j}}{j !}\left\{\frac{2\{\psi(3)-\ln [\lambda(j+2)]\}}{[\lambda(j+2)]^{3}}+\frac{\Gamma\left(\frac{1}{\beta}+3\right)\{\psi(2)-\ln [\lambda(j+2)]\}}{[\lambda(j+2)]^{\frac{1}{\beta}+3}}\right\}, \\
& I_{14}^{\prime}=I_{41}^{\prime}=-\frac{\lambda^{2} \beta \delta}{\alpha} \sum_{j=0}^{\infty} \frac{(-1)^{j}(\delta-2)_{j}}{j !}\left\{\frac{1}{[\lambda(j+2)]^{2}}+\frac{\Gamma\left(\frac{1}{\beta}+2\right)}{[\lambda(j+2)]^{\frac{1}{\beta}+2}}\right\}, \\
& I_{22}^{\prime}=-\frac{1}{\lambda^{2}}-2 \lambda \delta(\delta-1) \sum_{j=0}^{\infty} \frac{(-1)^{j}(\delta-3)_{j}}{j ![\lambda(j+2)]^{3}}, \\
& I_{23}^{\prime}=I_{32}^{\prime}=-\frac{\lambda \delta}{\beta} \sum_{j=0}^{\infty} \frac{(-1)^{j}(\delta-1)_{j}}{j !}\left\{\frac{\psi(2)-\ln [\lambda(j+1)]}{[\lambda(j+1)]^{2}}\right\} \\
& +\frac{\lambda \delta(\delta-1)}{\beta} \sum_{j=0}^{\infty} \frac{(-1)^{j}(\delta-2)_{j}}{j !}\left\{\frac{\psi(2)-\ln [\lambda(j+2)]}{[\lambda(j+2)]^{2}}\right\} \\
& -\frac{2 \lambda^{2} \delta(\delta-1)}{\beta} \sum_{j=0}^{\infty} \frac{(-1)^{j}(\delta-3)_{j}}{j !}\left\{\frac{\psi(3)-\ln [\lambda(j+2)]}{[\lambda(j+2)]^{3}}\right\} \text {, } \\
& I_{24}^{\prime}=I_{42}^{\prime}=\lambda \delta \sum_{j=0}^{\infty}\left\{\frac{(-1)^{j}(\delta-2)_{j}}{j ![\lambda(j+2)]^{2}}\right\} \text {, } \\
& I_{33}^{\prime}=-\frac{1}{\beta^{2}}-\frac{1 \lambda^{2} \delta}{\beta^{2}} \sum_{j=0}^{\infty} \frac{(-1)^{j}(\delta-1)_{j}}{j !}\left\{\frac{\{\psi(2)-\ln [\lambda(j+1)]\}^{2}+\zeta(2,2)}{[\lambda(j+1)]^{2}}\right\} \\
& +\frac{1 \lambda^{2} \delta(\delta-1)}{\beta^{2}} \sum_{j=0}^{\infty} \frac{(-1)^{j}(\delta-2)_{j}}{j !}\left\{\frac{\{\psi(2)-\ln [\lambda(j+2)]\}^{2}+\zeta(2,2)}{[\lambda(j+2)]^{2}}\right\} \\
& -\frac{2 \lambda^{3} \delta(\delta-1)}{\beta^{2}} \sum_{j=0}^{\infty} \frac{(-1)^{j}(\delta-3)_{j}}{j !}\left\{\frac{\{\psi(3)-\ln [\lambda(j+2)]\}^{2}+\zeta(2,3)}{[\lambda(j+2)]^{3}}\right\} \\
& I_{34}^{\prime}=I_{43}^{\prime}=\frac{1 \lambda^{2} \delta}{\beta} \sum_{j=0}^{\infty} \frac{(-1)^{j}(\delta-2)_{j}}{j !}\left\{\frac{\psi(2)-\ln [\lambda(j+2)]}{[\lambda(j+2)]^{2}}\right\},
\end{aligned}
$$


in which $\operatorname{Ei}(x), \psi(x)$ and $\zeta(x, q)$ are as given in (1.7), (1.8), (1.9) and (1.10).

\section{References}

Aarset, M. V. (1987), "How to identify bathtub hazard rate", IEEE Transactions on Reliability, $36,106-108$.

Almalki, S. J. and Nadarajah, S. (2014), "Modifications of the Weibull distribution: A review", Reliability Engineering and System Safety, 124, 32-55.

Bebbington, M., Lai, C. D. and Zitikis, R. (2007), “A flexible Weibull extension”, Reliability Engineering and System Safety, 92, 719-726.

Cordeiro, G. M., Nadarajah, S. and Ortega, M. M. (2013), "General results for the beta Weibull distribution", Journal of Statistical Computation and Simulation, 83, 1082-1114.

Famoye, F., Lee, C. and Olumolade, O. (2005), "The beta-Weibull distribution", Journal of Statistical Theory and Applications, 4, 121-136.

Gradshteyn, I. S. and Ryhzik, I. M. (2007), Tables of Integrals, Series and Products, Academic Press, New York.

Gupta, R. C., Gupta, P. L. and Gupta, R. D. (1998), "Modeling failure time data by Lehman alternatives", Communications in Statistics - Theory and Methods, 27, 887-904.

Henningsen, A. and Toomet, O. (2011), "maxLik: A package for maximum likelihood estimation in R", Computational Statistics, 26, 443-458.

Kumar, C. S. and Dharmaja, S. H. S. (2014), "On some properties of Kies distribution", Metron, 72, 97-122.

Kumar, C. S. and Dharmaja, S. H. S. (2016), "The Exponentiated Reduced Kies distributionProperties and Applications", Communications in Statistics-Theory and Methods, (published online).

Kumar, C. S. and Nair, B. U. (2012), "An alternative hyper-Poisson distribution”. Statistica, $72,357-369$.

Lee, E. T. and Wang, J. (2003), Statistical Methods for Survival Data Analysis, Wiley, New York.

Meeker, W. Q. and Escobar, L. A. (1998), Statistical Methods for Reliability Data, Wiley, New York.

Mudholkar, G. S., Srivastava, D. K. and Freamer, M. (1995), "The exponentiated Weibull family", Technometrics, 37, 436-445. 
Mudholkar, G. S. and Srivastava, D. K. (1993), "Exponentiated Weibull family for analyzing bathtub failure-rate data", IEEE Transactions on Reliability, 42, 299-302.

Murthy, D. N. P., Xie, M. and Jiang, R. (2004), Weibull Models, Wiley, New York.

Rinne, H. (2009), The Weibull Distribution: A Handbook, Taylor and Francis Group, London.

Singla, N., Jain, K. and Sharma, S. K. (2012), "The beta generalized Weibull distribution: Properties and applications", Reliability Engineering and System Safety, 102, 5-15.

Received: July 18, 2016

Accepted: May 17, 2017 